\title{
Designing a Multi-epitope Peptide Vaccine Against COVID-19 Variants Utilizing In-silico Tools
}

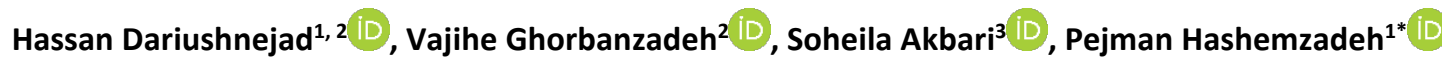

1. Department of Medical Biotechnology, Faculty of Medicine, Lorestan University of Medical Sciences, Khorramabad, Iran

2. Razi Herbal Medicines Research Center, Lorestan University of Medical Sciences, Khorramabad, Iran

3. Department of Obstetrics and Gynecology, Lorestan University of Medical Sciences, Khorramabad, Iran

\section{ABSTRACT}

Background and Aim: SARS-CoV-2 is the causative agent of Coronavirus 2019 or COVID-19 in the world. Novel coronavirus disease is a respiratory disease. To date, there have been challenges in the treatment for COVID-19 and emerged new variants like UK B1.1.7. Accordingly, an effective prevention regime is needed for this infection, which covers most variants. The purpose of this research was to predict the conserved epitopes of Spike and Nucleocapsid proteins from SARS-CoV-2 for the design of a novel coronavirus 2019 multi-epitope vaccine using in silico tools.

Materials and Methods: Computational analysis and immunoinformatics approaches include identification of potential conserve epitopes and selection of epitopes based on allergenicity, toxicity, antigenicity, and molecular docking were used for epitope prediction and screening. In the next step, selected segments of the epitopes were attached by the suitable linkers. Finally, Maltese-bound protein (MBP) as an adjuvant was added to the novel vaccine structure. The secondary and third structures of the designed multi-epitope vaccine were predicted via immunoinformatics algorithms. Predicted structure refined and validated for attaining best stability. In the end, immunoinformatics evaluation, molecular docking, and molecular dynamics were performed to confirm vaccine efficiency. Codon optimization and in silico cloning were done to ensure the expression yield of the novel multi-epitope vaccine in the target host.

Results: This study showed that our data support the suggestion that the designed vaccine could induce immune responses against SARS-CoV-2 variants.

Conclusion: The structure designed had acceptable quality with software reviews. Further in vitro and in vivo experiments are needed to confirm the safety and immunogenicity of the candidate vaccine.

Keywords: B1.1.7 variant, COVID-19, Immunoinformatics, SARS-CoV-2, Vaccine

Received: 2021/07/11; Accepted: 2021/09/01; Published Online: 2021/09/28

\begin{tabular}{|c|c|}
\hline Corresponding Information: & $\begin{array}{l}\text { Pejman Hashemzadeh, Department of Medical Biotechnology, School of Medicine, Kamalvand Campus, Lorestan University } \\
\text { of Medical Sciences, Khorramabad, Lorestan, Iran. Email:Pejman7genetian@gmail.com }\end{array}$ \\
\hline (c) (i) (9) & $\begin{array}{l}\text { This is an original open-access article distributed under the terms of the Creative Commons Attribution-n } \\
\text { distribution of the material just in noncommercial usages with proper citation. }\end{array}$ \\
\hline
\end{tabular}

Use your device to scan and read the article online

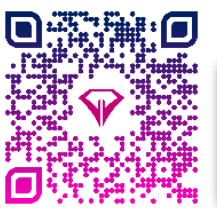

Dariushnejad H, Ghorbanzadeh V, Akbari S, Hashemzadeh P. Designing a Multi-epitope Peptide Vaccine Against COVID-19 Variants Utilizing In-silico Tools. Iran J Med Microbiol. 2021; 15 (5) :592605

\section{Download citation: BibTeX | RIS | EndNote | Medlars | ProCite | Reference Manager | RefWorks}

\section{Send citation to: $\otimes_{\text {Mendeley }} \mathbf{z}$ zotero $\underline{B}$ RefWorks}

\section{Introduction}

Coronaviridae is a family of viruses consist of seven members that have a single-stranded RNA with positive-sense, enveloped, and possess genome size of 17 to $91 \mathrm{~kb}$ (1). Four viruses from this family cause the common cold, and two of them lead to the deadly respiratory disease of severe acute respiratory syndrome (SARS) and middle east respiratory syndrome (MERS). The newly discovered seventh member of this family causes COVID-19 disease (2).

Based on genotyping and serology, coronaviruses are divided into alpha, beta, gamma and delta genus that alpha and beta genus caused health problems in human (3). In late 2019, a new beta coronavirus was isolated from people exposed to a seafood market in 
Wuhan, China, and named SARS-CoV-2 (4). Symptoms such as fever or chills, cough, shortness of breath or difficulty breathing, fatigue, muscle or body aches, headache, the new loss of taste or smell, sore throat, congestion or runny nose, nausea or vomiting, digestive problems, diarrhea, kidney failure among the main symptoms of COVID-19 (5-8).

SARS-CoV-2 spread globally, and the World Health Organization (WHO) and the Public Health Emergency of International Concern (PHEIC) declared a pandemic (9). After pandemic waves from Wuhan strain, a rapidly spreading variant emerged in the UK. This variant is derived from the SARS-CoV-2 20B/GR clade (lineage B.1.1.7). It contains multiple mutations, including a combination of the N501Y and the 6970del in Spike gene. These mutations caused increased transmissibility of the virus up to $71 \%$ over and above the previous circulating strains (10). Although a number of vaccines have been introduced to the community, it remains necessary to design a vaccine using conserve viral antigens, which cover current circulating variants.

SARS-CoV-2 possesses 14 open reading frames in its genome, including four structural proteins: the Spike protein $(S)$, the Envelope protein $(E)$, the membrane protein $(\mathrm{M})$, and the Nucleocapsid protein $(\mathrm{N})(11,12)$. Among these structural proteins, Spike proteins that reside on the virus surface are typically selected as antigens to produce antibodies from B-cells to neutralize the virus. Spike protein contains the receptor-binding domain (RBD) responsible for binding the ACE2 (angiotensin-converting enzyme 2) and entry into the cell. Therefore, the conserved sequence of $S$ protein is a major target antigen for vaccine development (13-17).

Nucleocapsid protein $(\mathrm{N})$ plays an important role in the viral cell cycle and contributes to forming helical ribonucleoproteins during the RNA genome packaging, regulate viral RNA synthesis during replication, transcription, and modulating metabolism in infected individuals. On the other hand, $\mathrm{N}$ gene is more conserved and stable. It is proved that high-level IgG against this protein secreted in sera of SARS patients, and the $\mathrm{N}$ protein is a representative antigen for the T-cell response in a vaccine setting $(18,19)$. The immune system, by its nature, can make its own adjustments to recognize vaccines and pathogens. Low immunogenicity is one of the drawbacks of vaccines. One approach to overcome this problem is to use the proper adjuvant (20).

Today, SARS-COV-2 mutations are one of the challenges in human populations immunization, so designing a vaccine that can target the conserved sequence of the virus is a new approach in this study. In the current study, with the aim of in silico immunoinformatic, a novel and common epitope of $\mathrm{S}$ and $\mathrm{N}$ proteins from COVID-19 significant variants predicted for designing multi-epitope vaccine against this global health problem.

\section{Materials and Methods}

2.1. Retrieval of protein sequences and alignment The FASTA format of surface glycoprotein (RefSeq: YP_009724390.1) and Nucleocapsid phosphoprotein (RefSeq: YP_009724397.2) of SARS-CoV-2 Wuhan strain were obtained from the National Center of Biotechnology Information NCBI at:

https://www.ncbi.nlm.nih.gov/protein/?term=SARSCoV-2 (21).

Multiple sequence alignment was performed through CLUSTALW at:

https://www.genome.jp/tools-bin/clustalw

\subsection{B-cell epitope (linear) prediction}

The Immune Epitope Database (IEDB) server at: http://tools.iedb.org/bcell/ includes data about immune epitopes for immune response system purposes. Bepipred linear epitope prediction server at http://www.cbs.dtu.dk/services/BepiPred/ was applied for predicting linear B cell epitopes based on target antigens-specific sequence features using amino acid and HMM scales $(22,23)$.

\subsection{Prediction of HLA class I and II epitopes} RANKEPEP server at:

http://imed.med.ucm.es/Tools/rankpep.html using Position Specific Scoring Matrices (PSSMs), Class I and Class II MHC molecules predicts the sequence of target antigens (24).

2.4. Allergenicity, toxicity and antigenicity prediction of the selected epitopes

The AllerTOP server at: https://www.ddgpharmfac.net/AllerTOP/ is a bioinformatics tool for predicting allergens. This server is the first server alignment-free that can predict whether or not target antigens are allergenic (25).

Server ToxinPred at:

http://crdd.osdd.net/raghava/toxinpred/is one of the best bioinformatics tools for predicting the toxicity of target antigens. The server for the detection from toxic epitopes of machine-learning technique support vector machine (SVM) is used (26).

Server VaxiJen at:

http://www.ddg-pharmfac.net/vaxijen/VaxiJen/VaxiJen.html is an in silico tool for predicting the antigenicity of target peptides. This server is the first server alignment-independent that can predict whether or not target peptides are protective antigens (27). 


\subsection{Generation of the 3D structures of the selected epitopes}

The PEP-FOLD 3 server at: https://bioserv.rpbs.univparis-diderot.fr/services/PEP-FOLD3/ is based on the structural description of peptides. In a short time, this server returns useful data in five top models.

\subsection{Molecular docking and refinement of the selected epitopes \\ PatchDock web servers at: \\ https://bioinfo3d.cs.tau.ac.il/PatchDock/php.php are} applied for molecular docking. This server is applied carry out structure prediction of protein-small and protein-protein molecule collections (28). Investigation of molecular docking of peptides using this server against alleles HLA DRB1*04-01 (PDB ID: 5JLZ) and HLA-A*11-01 allele (PDB ID: 5WJL) was done. PatchDock server algorithm is based on shape complementarity principles. This server uses the root mean square deviation (RMSD) score for candidate solutions. Top score solutions are considered the best solutions by the PatchDock server. In order to check the refinement and re-scoring of the docking results were carried out, we used FireDock server at: http://bioinfo3d.cs.tau.ac.il/FireDock/php.php

The server generates global energy for the best solutions and produces them based on global energy, and the lowest global energy is always considered the best docking score (29).

\subsection{Construction of final vaccine construct}

The potential epitopes of the two target antigens were selected and used in the final construction structure. After selecting the final epitopes, it is time to merge the epitopes into each other through the appropriate linkers GSGSGS, EAAAK and AAYKK. In this study, Maltese-bound protein (MBP) was used as adjuvants for the designed vaccine. This sequence is recognized by Toll Like Receptors (especially TLR2 and TLR4) and stimulates cellular and humoral immunity (30).

2.8. Evaluation of Antigenicity and allergenicity of recombinant vaccine

The antigenicity of the recombinant construction was specified using the ANTIGENpro server at: http://scratch.proteomics.ics.uci.edu/.

The allergenicity of the recombinant construction was determined using AllergenFP v1.0 server at: http://ddg-pharmfac.net/AllergenFP/.

2.9. Analysis of physicochemical properties of recombinant vaccines

In current study, ProtParam server at: https://web.expasy.org/protparam/ was used for calculating physicochemical properties of the novel vaccine, including molecular weight, theoretical pl (isoelectric point, El (extinction coefficient), R and $+R$ (total number of positive and negative residues), instability index II, GRAVY (grand average hydropathy) and $\mathrm{Al}$ (aliphatic index) (31).

2.10. Analysis of the secondary and tertiary structure of the recombinant vaccine

To predict the secondary structure, the GOR4 server at:

https://npsa-prabi.ibcp.fr/cgi-

bin/npsa_automat.pl?page=/NPSA/npsa gor4.html

was used. This server predicts the data theory of the second structure of the novel vaccine.

The three-dimensional structure of recombinant construct was predicted by Phyre 2 web server at: http://www.sbg.bio.ic.ac.uk/phyre2/html/page.cgi?id =index. Phyre2 server provides a useful tool to analyze and predict protein structure and function. This server is one of the best protein online prediction servers and works based on the hidden Markov model (HMM) (32). YASARA software was used for predicted models' visualization.

2.11. Refinement and validation of the third structure of the recombinant vaccine

In order to check the refinement of modeled 3D structure, we used GalaxyRefine at:

http://galaxy.seoklab.org/cgi-

bin/submit.cgi?type=REFINE. In the refined model, two servers, including RAMPAGE and ProsA, were used for validation.

RAMPAGE server at:

http://mordred.bioc.cam.ac.uk/ rapper/rampage.ph p calculate phi-psi torsion angles for each amino acid in the vaccine construct (33).

ProSA-web server at:

https://prosa.services.came.sbg.ac.at/prosa.php applied for structure validation and calculated an overall quality score (34).

\subsection{Molecular docking study}

Docking experiments were performed by the Z-Dock server (http://zdock.umassmed.edu/). This server carries out the prediction of interactive proteinprotein docking (35). The Cluspro server (https://cluspro.bu.edu/login.php) was used to evaluate the interaction between the recombinant vaccine and TLR4 / MD-2. On this server, vaccine protein was selected as ligand, and TLR4 / MD2 was introduced as a receptor for molecular docking (36).

\subsection{Molecular Dynamics Simulation}

We used the iMODS server at:

http://imods.chaconlab.org/ to check the stability of the protein complex. This server is a useful tool for molecular dynamics study. This server was used to explain the motion of a collective protein in internal coordinates through normal mode analysis (NMA). The server estimated the direction and extent of the 
immanent motions of the complex in terms of deformability, eigenvalues, B-factors, and covariance (37).

\subsection{Codon adaptation and in silico cloning}

SMS server at:

https://www.bioinformatics.org/sms/rev comp.html was used for reverse translation of the designed construct to the nucleotide sequence. In the next step, the Java Codon Adaptation tool (http://www.jcat.de/) was used for codon optimization to clone designed vaccine in Escherichia coli K12 strain expression host. Restriction sites BamHI and HindIII were introduced at the $\mathrm{N}$ and $\mathrm{C}$-terminal sites of the final construct for cloning in the pET 26+ expression vector.

\section{Results}

All steps taken to design a recombinant vaccine are shown in Figure 1.

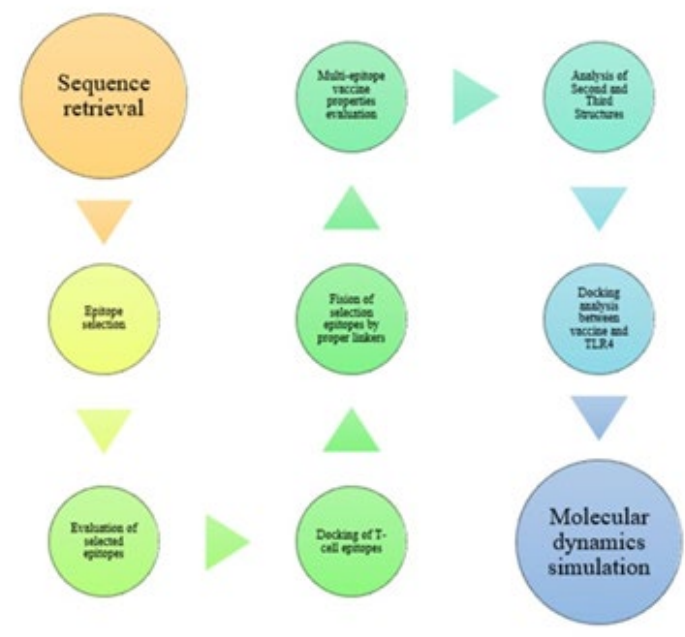

\subsection{B-cell binding epitopes}

Surface glycoprotein and nucleocapsid phosphoprotein sequences were used for B-cell binding epitopes prediction by Immune Epitope Database (IEDB) server (Table 1).

Table 1. B-cell peptide candidates

\begin{tabular}{llll} 
ANTIGENS & POS & SEQUENCE & SCORE \\
& 251 & PGDSSSG & 1.484 \\
Spike protein & 807 & PDPSKPS & 1.413 \\
& 252 & GDSSSGW & 1.404 \\
& 476 & GSTPCNG & 1.397 \\
Nucleocapsid & 2 & SDNGPQN & 1.439 \\
protein & 18 & GGPSDST & 1.417 \\
& 75 & NTNSSPD & 1.417 \\
\hline
\end{tabular}

\subsection{T-cell binding epitopes}

Surface glycoprotein and nucleocapsid phosphoprotein sequences were used for $\mathrm{MHCI}$ and MHC II binding epitopes prediction by the RANKEPEP server (Tables 2, 3).
Figure 1. Schematic procedure chart for designing the recombinant vaccine. The procedure used for designing the recombinant vaccine has briefly been shown.

Tables 2. MHC-I peptide candidates

\begin{tabular}{cccc}
\hline ANTIGENS & POS & SEQUENCE & SCORE \\
& 154 & ESEFRVYSS & 19.406 \\
Spike protein & 196 & NIDGYFKIY & 16.09 \\
& 661 & ECDIPIGAG & 13.93 \\
& 576 & VRDPQTLEI & 13.088 \\
\hline Nucleocapsid & 1180 & QKEIDRLNE & 12.734 \\
protein & 295 & GTDYKHWPQ & 15.609 \\
& 103 & DLSPRWYFY & 13.047 \\
\hline
\end{tabular}


Table 3. MHC-II peptide candidates

\begin{tabular}{cccc}
\hline ANTIGENS & POS & SEQUENCE & SCORE \\
& 144 & YYHKNNKSW & 19.71 \\
Spike protein & 199 & GYFKIYSKH & 17.658 \\
& 1208 & QYIKWPWYI & 17.142 \\
& 832 & GFIKQYGDC & 16.276 \\
& 169 & EYVSQPFLM & 15.561 \\
Nucleocapsid & 955 & NAQALNTLV & 15.311 \\
protein & 896 & FAMQMAYRF & 13.314 \\
& 365 & YSVLYNSAS & 12.812 \\
& 86 & YYRRATRRI & 27.321 \\
\hline
\end{tabular}

\subsection{Allergenicity, toxicity and antigenicity determination}

T-cell and B-cell epitopes that were selected by RANKEPEP and IEDB servers have been evaluated for allergenicity, toxicity, and antigenicity by mentioned servers in the material and method section (Tables 4). In the next step, ESEFRVYSS, VRDPQTLEI, DLSPRWYFY

and QAFGRRGPPE epitopes were selected from MHC Class I, and EYVSQPFLM, FAMQMAYRF and RQKKQQTVT epitopes were selected from MHC Class II and PGDSSSG, GDSSSGW and GSTPCNG epitopes were selected from B-cell.

Table 4. Allergenicity, antigenicity, and toxicity analysis of the selected T-cell and B-cell epitopes of Spike protein and Nucleocapsid protein.

\begin{tabular}{|c|c|c|c|c|}
\hline ANTIGENS & EPITOPES & ANTIGENCITY & ALLERGENCITY & TOXICITY \\
\hline \multirow{17}{*}{ Spike protein } & PGDSSSG & ANTIGEN & NON-ALLERGEN & NON-TOXIN \\
\hline & PDPSKPS & NON-ANTIGEN & NON-ALLERGEN & NON-TOXIN \\
\hline & GDSSSGW & ANTIGEN & NON-ALLERGEN & NON-TOXIN \\
\hline & GSTPCNG & ANTIGEN & NON-ALLERGEN & NON-TOXIN \\
\hline & ESEFRVYSS & ANTIGEN & NON-ALLERGEN & NON-TOXIN \\
\hline & NIDGYFKIY & NON-ANTIGEN & ALLERGEN & NON-TOXIN \\
\hline & ECDIPIGAG & ANTIGEN & ALLERGEN & NON-TOXIN \\
\hline & VRDPQTLEI & ANTIGEN & NON-ALLERGEN & NON-TOXIN \\
\hline & QKEIDRLNE & NON-ANTIGEN & NON-ALLERGEN & NON-TOXIN \\
\hline & YYHKNNKSW & NON-ANTIGEN & NON-ALLERGEN & NON-TOXIN \\
\hline & GYFKIYSKH & NON-ANTIGEN & ALLERGEN & NON-TOXIN \\
\hline & QYIKWPWYI & ANTIGEN & ALLERGEN & NON-TOXIN \\
\hline & GFIKQYGDC & NON-ANTIGEN & ALLERGEN & NON-TOXIN \\
\hline & EYVSQPFLM & ANTIGEN & NON-ALLERGEN & NON-TOXIN \\
\hline & NAQALNTLV & ANTIGEN & ALLERGEN & NON-TOXIN \\
\hline & FAMQMAYRF & ANTIGEN & NON-ALLERGEN & NON-TOXIN \\
\hline & YSVLYNSAS & NON-ANTIGEN & ALLERGEN & NON-TOXIN \\
\hline \multirow{7}{*}{$\begin{array}{c}\text { Nucleocapsid } \\
\text { protein }\end{array}$} & SDNGPQN & ANTIGEN & NON-ALLERGEN & NON-TOXIN \\
\hline & GGPSDST & ANTIGEN & NON-ALLERGEN & NON-TOXIN \\
\hline & NTNSSPD & ANTIGEN & NON-ALLERGEN & NON-TOXIN \\
\hline & NSTPGSS & ANTIGEN & NON-ALLERGEN & NON-TOXIN \\
\hline & GTDYKHWPQ & NON-ANTIGEN & ALLERGEN & NON-TOXIN \\
\hline & DLSPRWYFY & ANTIGEN & NON-ALLERGEN & NON-TOXIN \\
\hline & AADLDDFSK & NON-ANTIGEN & NON-ALLERGEN & NON-TOXIN \\
\hline
\end{tabular}




\begin{tabular}{c|ccc|c}
\hline ANTIGENS & EPITOPES & ANTIGENCITY & ALLERGENCITY & TOXICITY \\
\hline QAFGRRGPE & ANTIGEN & NON-ALLERGEN & NON-TOXIN \\
\hline YYRRATRRI & NON-ANTIGEN & NON-ALLERGEN & NON-TOXIN \\
\hline GYYRRATRR & NON-ANTIGEN & NON-ALLERGEN & NON-TOXIN \\
\hline RQKKQQTVT & ANTIGEN & NON-ALLERGEN & NON-TOXIN \\
KKQQTVTLL & NON-ANTIGEN & NON-ALLERGEN & NON-TOXIN \\
KGQQQQGQT & ANTIGEN & ALLERGEN & NON-TOXIN \\
\hline
\end{tabular}

\subsection{Generation of three-dimensional structures of epitopes and molecular docking}

In this study, molecular docking was used to determine whether the final epitopes were able to bind to MHC class I and class II. For docking study, selected MHC class 1 and 2 epitopes were introduced to the server as ligand, and the HLA-A*11-01 allele (PDB ID: 5WJL) and HLA DRB1*04-01 (PDB ID: 5JLZ) were presented as the receptor. Amongst MHC-I and
MHC-II epitopes from surface glycoprotein, ESEFRVYSS and EYVSQPFLM demonstrate the outcome with the lowest and best global energy score. Amongst MHC-I and MHC-II epitopes of nucleocapsid phosphoprotein, DLSPRWYFY and RQKKQQTVT demonstrate the best outcome with the lowest and highest global energy score (Table 5).

Table 5. Results of molecular docking for T-cell epitope against two HLA types

\begin{tabular}{ccccc} 
Name of the protein & Epitope & MHC allele & Global energy & Hydrogen bond energy \\
\cline { 2 - 4 } & ESEFRVYSS & HLA-A*11-01 & -29.75 & -2.56 \\
\cline { 2 - 4 } Spike protein & VRDPQTLEI & HLA-A*11-01 & -22.86 & -2.18 \\
& EYVSQPFLM & HLA DRB1*04-01 & -18.27 & -4.95 \\
\cline { 2 - 4 } & FAMQMAYRF & HLA DRB1*04-01 & -7.96 & -2.12 \\
\multirow{2}{*}{$\begin{array}{c}\text { Nucleocapsid } \\
\text { protein }\end{array}$} & DLSPRWYFY & HLA-A*11-01 & -48.03 & -2.83 \\
& QAFGRRGPE & HLA-A*11-01 & -9.09 & -1.31 \\
\hline
\end{tabular}

\subsection{Epitope selection and construction of the multi-epitope peptide vaccine}

Based on high-ranking T-cell and B-cell epitopes, fourteen epitopes from two antigens were selected as the final regions. The final epitopes of each antigen were fused together by GSGSGS and AAYKK linkers. Maltose / maltodextrin-binding protein was also added as an adjuvant to the N-terminal of the novel vaccine with Linker EAAAK. The designed novel vaccine construct consisted of 590 amino acid residues, as illustrated in Figure 2.

a)

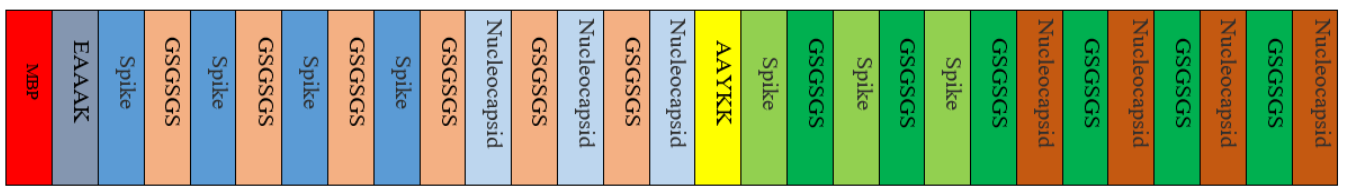

b)
HPDKLEEKFPQVAATGDGPDIIFWAHDRFGGYAQSGLLAEITPDKAFQDKLYPFTWDAVRYNGK
LIAYPIA VEALSLIYNKDLLPNPPKTWEEIPALDKELKAKGKSALMFNLQEPYFTWPLIAADGGY
AFKYENGKYDIKDVGVDNAGAKAGLTFLVDLIKNKHMNADTDYSIAEAAFNKGETAMTINGP
WAWSNIDTSKVNYGVTVLPTFKGQPSKPFVGVLSAGINAASPNKELAKEFLENYLLTDEGLEAV
NKDKPLGAVALKSYEEELAKDPRIAATMENAQKGEIMPNIPQMSAFWYAVRTAVINAASGRQT
VDEALKDAQTRITKEAAAKESEFRVYSSGSGSGSVRDPQTLEIGSGSGSEYVSQPFLMGSGSGSF
AMQMAYRFGSGSGSDLSPRWYFYGSGSGSQAFGRRGPEGSGSGSRQKKQQTVTAAYKKPGDSS
SGGSGSGSGDSSSGWGSGSGSGSTPCNGGSGSGSSDNGPQNGSGSGSGGPSDSTGSGSGSNTNSS
PDGSGSGSNSTPGSS

Figure 2. a) Graphical illustration of the multi-epitope vaccine construct. b) The sequence comprises 590 amino acid sequences. The vaccine contains an MBP adjuvant at the $\mathrm{N}$-terminal of vaccine construct 


\subsection{Evaluation of antigenicity and allergenicity of recombinant vaccine construct}

The results of the AllergenFP 1.0 server showed that the designed structure was not an allergen. The possibility of the designed recombinant construct

antigenicity was predicted 0.909 by the ANTIGENpro server, which means that our novel vaccine can stimulate impressive immune system responses.

\subsection{Analysis of physicochemical properties of recombinant vaccines construct}

The physicochemical parameters of the recombinant construct include GRAVY, theoretical pl, half-life, instability index, aliphatic index, amino acid composition, and molecular weight are shown in Table 6.

Table 6. Prediction results of recombinant vaccine physicochemical parameters

\begin{tabular}{lll}
\hline Physicochemical properties & Result \\
\hline GRAVY & -0.453 \\
\hline theoretical pl & 5.50 & \\
\hline & The estimated half-life is: 30 hours (mammalian reticulocytes, in vitro). \\
half-life & & $>$ 20 hours (yeast, in vivo). \\
& $>10$ hours (Escherichia coli, in vivo). \\
\hline instability index & 26.44 & \\
\hline aliphatic index & 63.27 & \\
\hline Total number of negatively charged residues & 64 & \\
\hline Total number of positively charged residues & 58 \\
\hline molecular weight & 61854.64 \\
\hline
\end{tabular}

\subsection{Secondary and tertiary structure analysis}

GOR IV server predictions show that our recombinant vaccine was composed of $33.90 \%$ alpha helix $(\mathrm{H}), 15.08 \%$ extended strand, and $51.02 \%$ random coil (C) secondary structural elements. Also, the three-dimensional model of our novel vaccine is produced by the phyre 2 server and is shown in Figure 3. The Phyre2 web server showed three main domains in the third structure of the designed vaccine. This server indicated that 369 residues $(63 \%$ of vaccine sequence) had been modeled with $100.0 \%$ confidence by the single highest scoring template.

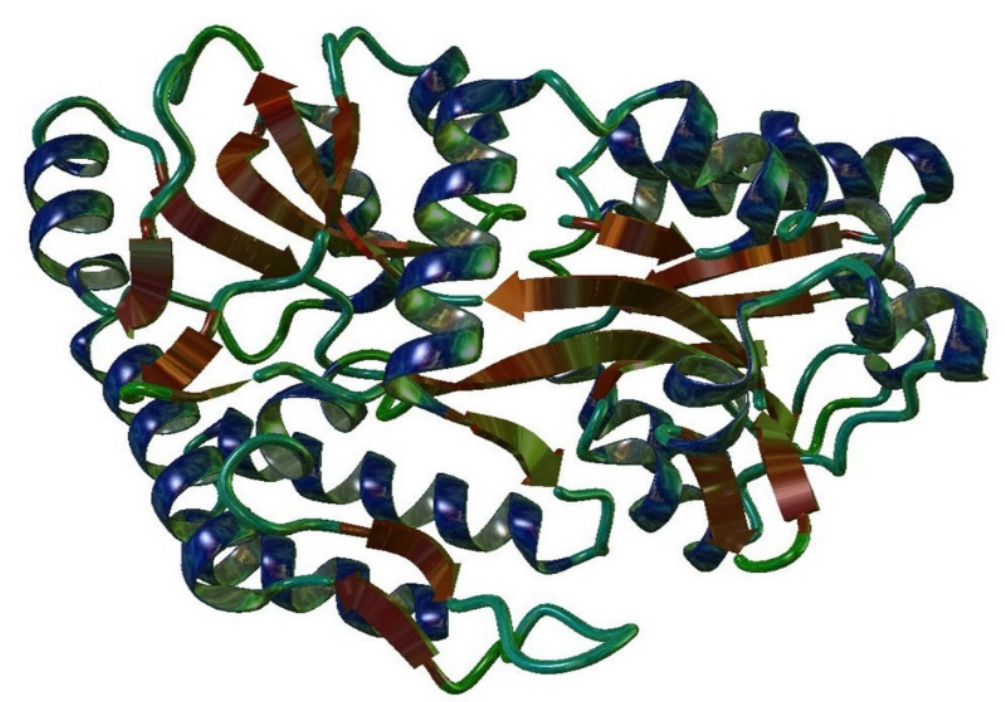

Figure 3. The predicted 3D structures of the recombinant vaccine generated by the Pyre2 server. 3D structure of protein vaccine structures generated by YASARA software.

\subsection{Tertiary structure refinement and validation}

The selected model was refined using GalaxyRefine. This server introduced five refined three-dimensional models. The first model with the highest score was selected and displayed in Figure 4. 


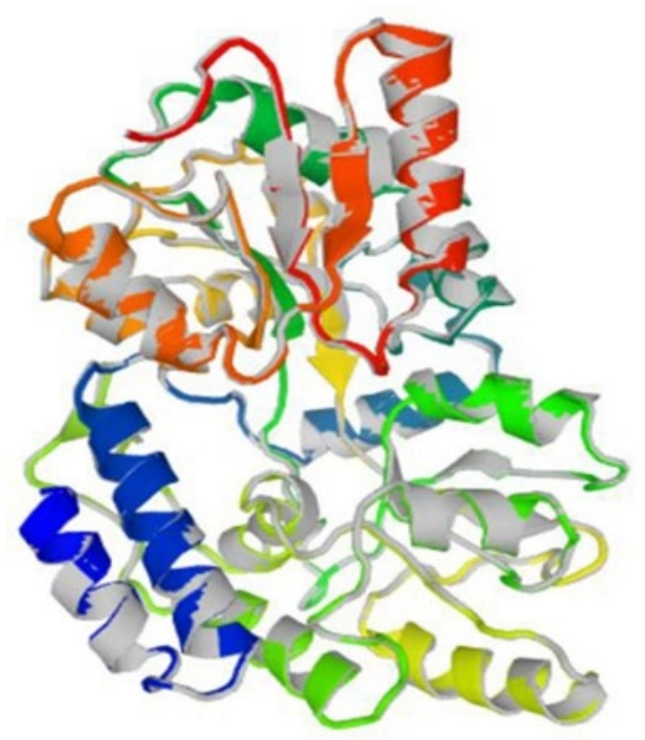

Figure 4. Final vaccine construct after refinement via GalaxyRefine server. The server genertaed five refined three-dimensional models. The first model with the highest score was selected and visulized by YASARA software.
The refined novel vaccine structures were validated with the aid of the ProSA-web and RAMPAGE servers. The ramachandran plot server showed that the recombinant vaccine had $99.2 \%$ number of residues in the favour region and $0.8 \%$ number of residues in the allowed region (Figure 5a). ProSA-web servers were used for the evaluation of potential and quality errors in the 3D crude model. This server is utilized for the prediction of Z-score prediction, which is found as -11.91 (Figure 5b).
A

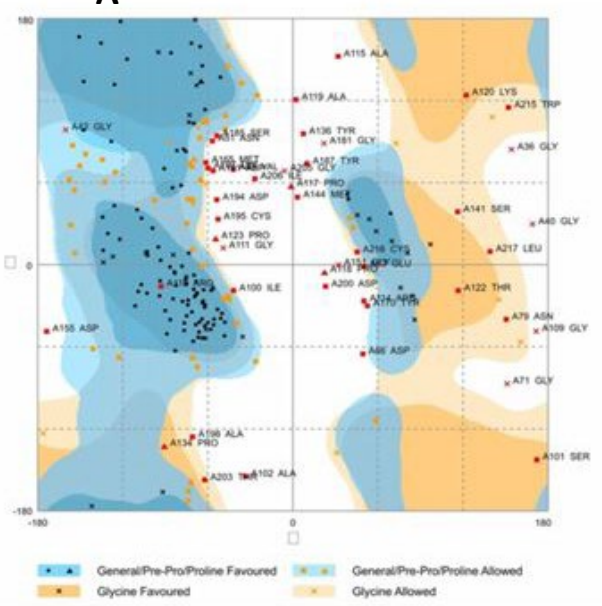

B

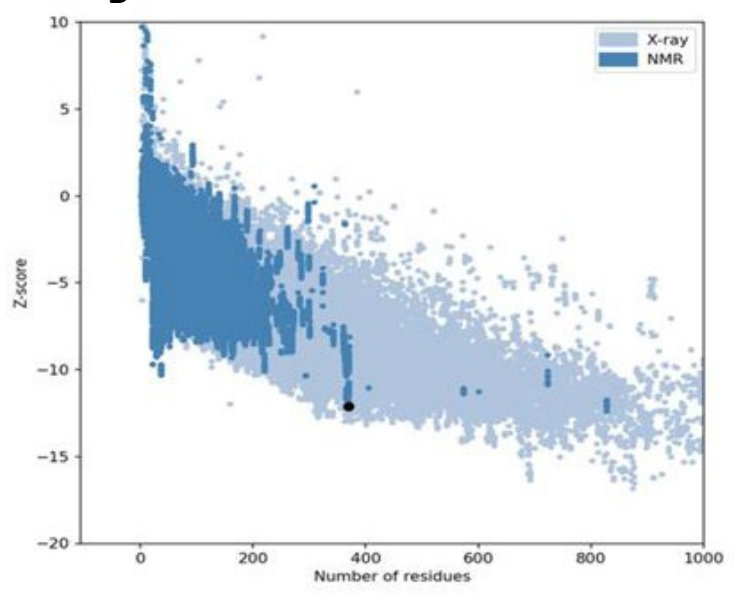

Figure 5. a) Ramachandran plot generated by RAMPAGE server. Ramachandran diagram showed that the recombinant vaccine had $99.2 \%$ number of residues in the favored region and $0.8 \%$ number of residues in the allowed region. b) ProSA-web server was used for the evaluation of potential and quality errors in 3D crude model. The Z-score of the constructed model calculated -11.91 , which is in the range of native protein conformation scores.

\subsection{Molecular docking of subunit vaccine with TLR-4}

A novel multi epitopes vaccine was docked with TLR4 using the Z-DOCK server. In general, ten complexes have been developed, and the most appropriate set of TLR vaccines has been selected based on the correct combination and binding (Figure 6a). The Cluspro server was also used to evaluate the interaction between the recombinant vaccine and
TLR4 / MD-2. The top 10 models were selected based on the biophysical characteristics of the receptor and ligand, which in terms of the docking pattern between the receptor and the ligand, the first introduced model possess the lowest weight score $(-915.9 \mathrm{kcal} / \mathrm{mol})$ (Figure 6b). 
a

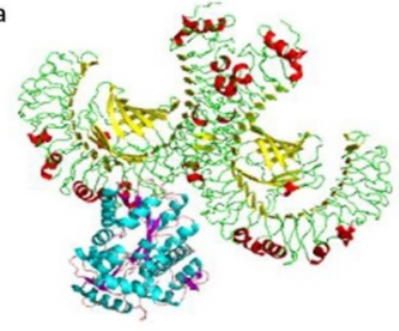

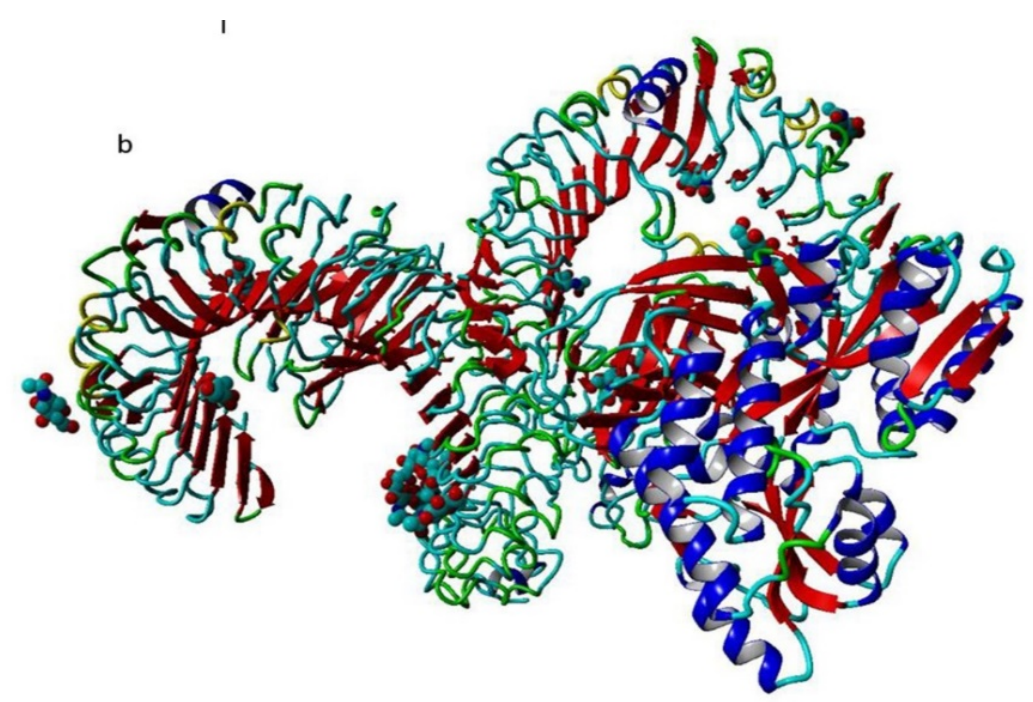

Figure 6. Docking studies of designed multi-epitope vaccine and modeled TLR4. a) Docked complex of TLR4 and multi-epitope vaccine generated by Z-DOCK server. b) Cluspro server used to generate binding sites of multi-epitope vaccine with TLR4.

\subsection{Molecular dynamic simulation}

Natural state analysis (NMA), large-scale flexibility, and stabilization of the recombinant vaccine were investigated by the iMOD server. This server is relevant to the internal coordinates of the docked complex. Figure 7a shows the probable deformity of the vaccine-TLR-4 related to the individual distortion of any residue shown by the chain hinges (green). Figure $7 \mathrm{~b}$ shows the B-factor values equivalent to the RMS inferred via NMA. Figure 7c shows the eigenvalue for the complex, equal to 1.922. Figure $7 d$ demonstrated the colored bars of the cumulative (green) and individual (red) variances that are inversely related to the eigenvalue. Figure $7 e$ shows the coupling between the different interactions of the residues by the covariance matrix, i.e., uncorrelated to white, red-correlated, and anti-correlation with blue. Figure $7 f$ shows the elastic network model that distinguishes pairs of atoms associated with springs.
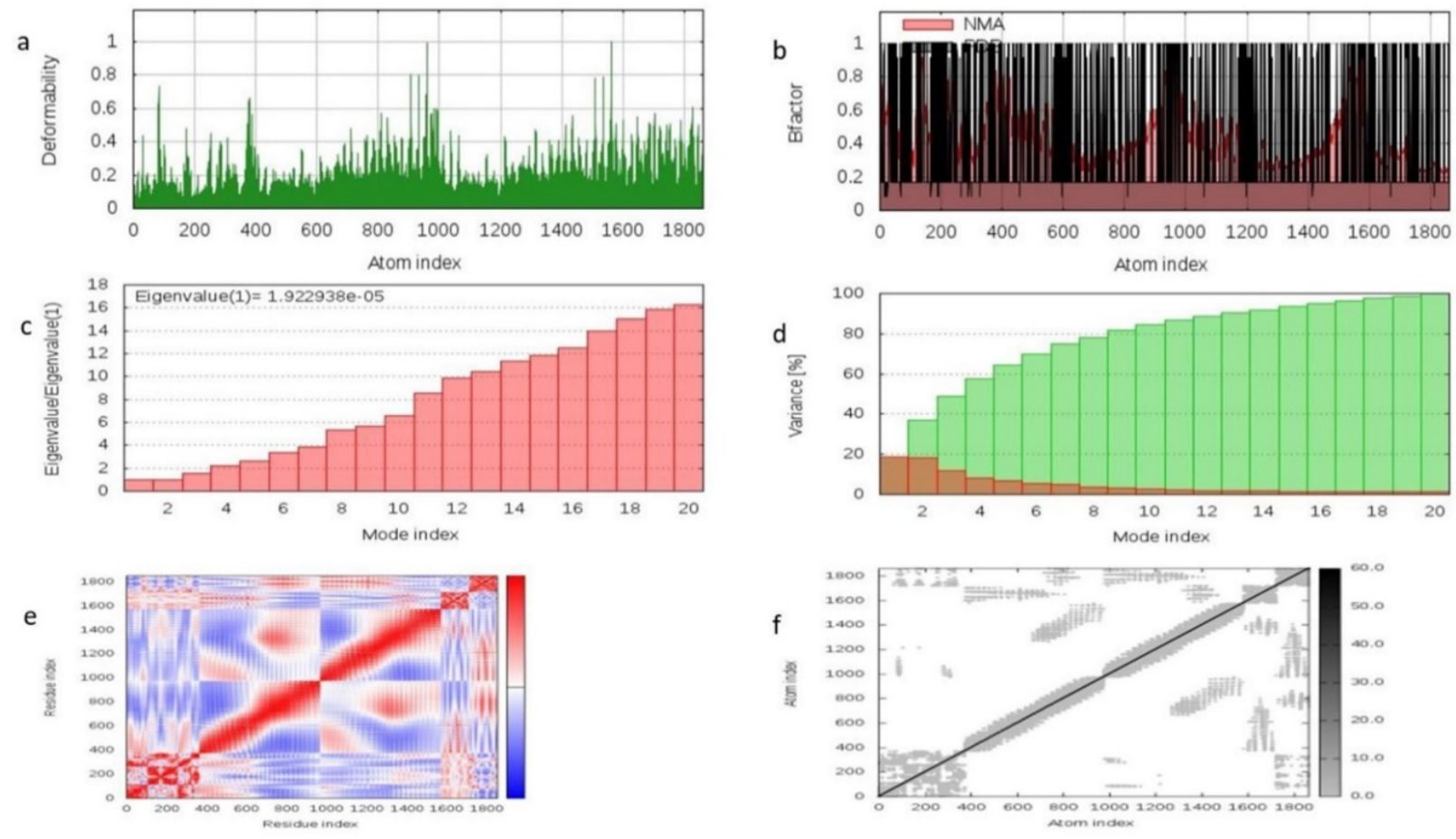

Figure 7. Schematic displaying the findings of MD simulation study of TLR-4 and recombinant vaccine docked complex. Here, (a) eigenvalues, (b) B-factor, (c) deformability, (d) variances, (e) covariance and (f) elastic network analysis. 


\subsection{Codon optimization and in silico cloning}

The SMS server conducted back translation of the vaccine construct. JCAT server was used to assess the key properties of the vaccine DNA sequence, including the GC content and Codon Adaptation Index (CAI). The CAI value for the optimized nucleotide sequence was 1 , and the GC content of the optimized sequence was calculated as $50.11 \%$ in E. coli. These outcomes suggest that the optimized vaccine DNA sequence will have a maximum expression yield in $E$. coli.

\section{Discussion}

The number of people who get infected by COVID19 diseases has increased day by day, as well as the increase in mortality that has caused international concern (38). Therefore, vaccination is an effective way to prevent COVID-19 diseases (39).

In this research, we determined the epitopes of potentially immune cells of two SARS-CoV-2 antigens and designed a multi-epitopes vaccine against current variants COVID-19. The SARS-CoV-2 antigenic proteins were evaluated by using in-silico tools, and then the antigenic parts of the Nucleocapsid and Spike proteins were selected for use in the novel vaccine. Immunoinformatic approaches are novel strategies for identifying, designing, and manufacturing specific antigen epitopes for use in a variety of purposes such as; vaccines, immunotherapy, etc. (40).

The novel multi-epitope vaccines are designed to stimulate the immunity of specific pathogens by selectively stimulating specific B-cell and T-cell antigens (41). In comparison with viruses that posse's DNA genetic material, viruses that contain RNA genetic material such as SARS-CoV-2 have a greater tendency to mutation. These mutations in this virus occur because of the lack of proofreading activity of polymerases, and this phenomenon causes resistance to drugs and escapes from immune surveillance (4244). These mutations led to emerging of new variants, of which the UK B1.1.7 strain is one. This strain possesses transmissibility of up to $71 \%$ over, increased clinical severity of illness, and vaccine escape capability (10).

Spike protein has two important regions with conserved sequences, include the receptor-binding domain (RBD) and N-terminal domain (NTD). These two regions are characterized by surface exposure and high antigenicity, and these two regions are outside of the area prone to mutations (45). In contrast, the Nucleocapsid protein is more conserved and stable, with $90 \%$ amino acid homology and fewer mutations over time. Nucleocapsid proteins of many coronaviruses are expressed abundantly during infection and are highly immunogenic (46). It was reported that high levels of IgG antibodies against $\mathrm{N}$ protein were secreted in the sera of SARS patients
(47). In SARS vaccine design, N protein was used for Tcell proliferation, response, and cytotoxic activity (48, 49).

The COVID-19 multi-epitope vaccine design was performed by in silico tools in several steps, includes immunoinformatic analysis, selection of conserved epitopes and multi-epitopes vaccine construction, immunoinformatic evaluation, analysis of secondary and third structures and study of the interaction between the novel vaccine and ligand (TLR-4). An effective vaccine must have both T-cell epitope and Bcell epitope to stimulate cellular and humoral immunity against pathogens (41). Appropriate servers were used to predict epitopes of T-cells and B cells. There are two main types of cells that have functions in the immune system, including T lymphocytes and B lymphocytes (50). The epitopes of T-cell (MHC-I, MHCII) and B-cell were identified by the servers of RANKEPEP and IEDB, respectively. High-scoring and potential epitopes (T-cell and B-cell epitopes) with non-toxicity, non-allergenicity, and antigenicity were selected for recombinant vaccine construction.

Then, the PEP-FOLD server generates threedimensional structures of MHC class-I epitopes and MHC class-II epitopes to study molecular docking. All selected T-cell epitopes have acceptable molecular docking scores, so it can be concluded that all selected epitopes have the ability to bind to their respective targets and stimulate an immune response in a robust manner.

After studying the docking of T-cell epitopes, the design of the recombinant construction was performed. An adjuvant is an essential component of the novel human vaccines (51). These compounds are used to increase the immunogenicity of vaccines, including DNA vaccines and multi-epitope vaccines (52). Therefore, combining adjuvants with protein epitopes can effectively stimulate specific antigenspecific immune responses (53). MBP, a protein with a molecular weight of $42 \mathrm{kDa}$, is responsible for binding and transporting maltose from the periplasmic space of bacteria of the Gram-negative model. MBP has been reported to act as an adjunct to specific immunity through specific receptors. It has also been reported that this protein increases lymphocytes (5456). In this recombinant vaccine construct, MBP was used as an adjuvant. On the other hand, MBP also can facilitate the expression and purification process of recombinant vaccine in $E$. coli, by one-step purification using affinity chromatography (57).

The linker is an important component in fusion epitopes to the design of a recombinant construction. Based on the structure of the linkers, the linkers are divided into 3 types, which include: flexible linkers, 
rigid linkers, and cleavable linkers. Hare, GSGSGS flexible linker and AAYKK cleavable linker were used for fusion between the epitopes, and the EAAAK rigid linker was used for fusion between the adjuvant and the epitope (58).

After vaccine construct design, the allergenicity, toxicity, antigenicity, and physiochemical analysis revealed that the designed vaccine was stable and possessed proper immunogenicity. Moreover, molecular docking and molecular dynamics simulations indicate that the designed vaccine has stable interaction with TLR4 ligand.

In the end, codon adaptation and in silico cloning experiments were performed to ensure that vaccine efficiently express in the target host, $E$. coli strain K12. $(59,60)$.

\section{Conclusion}

COVID-19 pandemic is the deadliest outbreak in recent decades. Prevention of the newly emerging novel coronavirus infection and mutated form of that is a very challenging problem for global health. In silico tools can help us to conquer this problem by saving time and cost. In this study, immunoinformatic and reverse vaccinology help us design a potential multiepitope vaccine against this new virus. Various in silico and computational studies of the proposed vaccine constructs indicate that this multi-epitope vaccine might confer proper immunogenic response. With complementary experimental and clinical studies, the

\section{References}

1. Payne S. Family Coronaviridae. Viruses. 2017:149. [DOI:10.1016/B978-0-12-803109-4.00017-9]

2. Al-Rohaimi AH, Al Otaibi F. Novel SARS-CoV-2 outbreak and COVID19 disease; a systemic review on the global pandemic. GENES DIS. 2020. [DOI:10.1016/i.gendis.2020.06.004] [PMID] [PMCID]

3. Ye Z-W, Yuan S, Yuen K-S, Fung S-Y, Chan C-P, Jin $D-Y$. Zoonotic origins of human coronaviruses. Int. J. Biol. Sci. 2020;16(10):1686. [DOI:10.7150/ijbs.45472] [PMID] [PMCID]

4. Sun $P, \operatorname{Lu} X, X u C$, Sun $W$, Pan B. Understanding of COVID-19 based on current evidence. J. Med. Virol. 2020;92(6):548-51. [DOI:10.1002/jmv.25722] [PMID] [PMCID]

5. Song $Y$, Liu $P$, Shi $X$, Chu $Y$, Zhang J, Xia J, et al. SARS-CoV-2 induced diarrhoea as onset symptom in patient with COVID-19. Gut. 2020;69(6):11434. [DOI:10.1136/gutjnl-2020-320891] [PMID] current study should be helpful to researchers to develop a potential vaccine against SARS-CoV-2 virus variants.

\section{Acknowledgment}

This research was funded by the Deputy of Research and Technology, Lorestan University of Medical Sciences, Khorramabad, Iran (grant NO. 1397-1-991345). The authors appreciate this financial support

\section{Conflict of Interest}

All authors declared that there is no conflict of interest.

\section{Funding}

This research was financially supported by Lorestan University of Medical Sciences, Khorramabad, Iran. Hereby the authors appreciate all the people who helped in this research.

\section{Ethics approval}

The present study was approved by The Ethics Committee of Lorestan University of Medical Sciences (IR.LUMS.REC.1398.293). This article does not contain any studies with human participants or animals performed by any of the authors.

6. Bouaziz J, Duong $\mathrm{T}$, Jachiet $\mathrm{M}$, Velter $\mathrm{C}$, Lestang $\mathrm{P}$, Cassius $\mathrm{C}$, et al. Vascular skin symptoms in COVID19: a french observational study. J. Eur. Acad. Dermatol. Venereol. 2020. [DOI:10.1111/jdv.16544] [PMCID]

7. Struyf T, Deeks JJ, Dinnes J, Takwoingi $\mathrm{Y}$, Davenport C, Leeflang MM, et al. Signs and symptoms to determine if a patient presenting in primary care or hospital outpatient settings has COVID-19 disease. Cochrane Database Syst. Rev. 2020(7). [DOI:10.1002/14651858.CD013665] [PMID] [PMCID]

8. Yang Z, Bogdan P, Nazarian S. An in silico deep learning approach to multi-epitope vaccine design: a SARS-CoV-2 case study. Sci. Rep. 2021;11(1):1-21. [DOI:10.1038/s41598-02181749-9] [PMID] [PMCID]

9. Jebril N. World Health Organization declared a pandemic public health menace: A systematic 
review of the coronavirus disease 2019 "COVID19", up to 26th March 2020. Available at SSRN 3566298. 2020. [DOI:10.2139/ssrn.3566298]

10. Tang JW, Tambyah PA, Hui DS. Emergence of a new SARS-CoV-2 variant in the UK. J. Infect. 2020. [DOI:10.1016/i.jinf.2020.12.024] [PMID] [PMCID]

11. Boopathi S, Poma AB, Kolandaivel P. Novel 2019 coronavirus structure, mechanism of action, antiviral drug promises and rule out against its treatment. J. Biomol. Struct. Dyn. 2020:1-10. [DOI:10.1080/07391102.2020.1758788] [PMID] [PMCID]

12. Pathak SK. General Details of Structural Proteins of Coronaviruses with Special Reference of SARSCOV-2 or COVID-19. Bull. Env. Pharmacol. Life Sci. 2020;9:34-8.

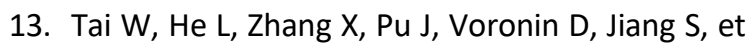
al. Characterization of the receptor-binding domain (RBD) of 2019 novel coronavirus: implication for development of RBD protein as a viral attachment inhibitor and vaccine. Cell. Mol. Immunol. 2020;17(6):613-20. [PMID] [PMCID] [DOI:10.1038/s41423-020-0400-4]

14. Chen $\mathrm{W}-\mathrm{H}$, Hotez PJ, Bottazzi ME. Potential for developing a SARS-CoV receptor-binding domain (RBD) recombinant protein as a heterologous human vaccine against coronavirus infectious disease (COVID)-19. Hum. Vaccines Immunother. 2020:1-4. [PMID] [PMCID] [DOI:10.1080/21645515.2020.1740560]

15. Choi J-H, Woo H-M, Lee T-y, Lee S-y, Shim S-M, Park W-J, et al. Characterization of a human monoclonal antibody generated from a B-cell specific for a prefusion-stabilized spike protein of Middle East respiratory syndrome coronavirus. PLoS One. 2020;15(5):e0232757. [PMID] [PMCID] [DOI:10.1371/journal.pone.0232757]

16. Chen X, Li R, Pan Z, Qian C, Yang Y, You R, et al. Human monoclonal antibodies block the binding of SARS-CoV-2 spike protein to angiotensin converting enzyme 2 receptor. Cell. Mol. Immunol. 2020:1-3. [DOI:10.1038/s41423-0200426-7] [PMID] [PMCID]

17. Kumar S. Drug and vaccine design against Novel Coronavirus (2019-nCoV) spike protein through Computational approach. Preprints. 2020. [DOI:10.20944/preprints202002.0071.v1]

18. Thieme $C$, Anft M, Paniskaki K, Blázquez Navarro A, Doevelaar A, Seibert FS, et al. The SARS-CoV-2 T-cell immunity is directed against the spike, membrane, and nucleocapsid protein and associated with COVID 19 severity. Cell Rep Med. 2020. [DOI:10.2139/ssrn.3606763]
19. Dutta NK, Mazumdar K, Gordy JT. The nucleocapsid protein of SARS-CoV-2: a target for vaccine development. J. Virol. 2020;94(13). [DOI:10.1128/JVI.00647-20]

20. Hashemzadeh P, Ghorbanzadeh V, Lashgarian HE, Kheirandish F, Dariushnejad H. Harnessing Bioinformatic Approaches to Design Novel Multiepitope Subunit Vaccine Against Leishmania infantum. nt J Pept Res Ther. 2019:1-12. [DOI:10.1007/s10989-019-09949-6]

21. Pruitt KD, Tatusova T, Maglott DR. NCBI reference sequences (RefSeq): a curated non-redundant sequence database of genomes, transcripts and proteins. Nucleic Acids Res. 2007;35(suppl_1): D61-D5. [DOI:10.1093/nar/gkl842] [PMID] [PMCID]

22. Larsen JEP, Lund O, Nielsen M. Improved method for predicting linear B-cell epitopes. Immunome Res. 2006;2(1):2. [DOl:10.1186/1745-7580-2-2] [PMID] [PMCID]

23. Ponomarenko JV, Bourne PE. Antibody-protein interactions: benchmark datasets and prediction tools evaluation. BMC Struct. Biol. 2007;7(1):64. [DOI:10.1186/1472-6807-7-64] [PMID] [PMCID]

24. Reche PA, Glutting J-P, Zhang H, Reinherz EL. Enhancement to the RANKPEP resource for the prediction of peptide binding to $\mathrm{MHC}$ molecules using profiles. Immunogenetics. 2004;56(6):40519. [DOI:10.1007/s00251-004-0709-7] [PMID]

25. Dimitrov I, Flower DR, Doytchinova I, editors. AllerTOP-a server for in silico prediction of allergens. BMC Bioinform. 2013. [PMID] [PMCID] [DOI:10.1186/1471-2105-14-S6-S4]

26. Gupta S, Kapoor P, Chaudhary K, Gautam A, Kumar R, Raghava GP, et al. In silico approach for predicting toxicity of peptides and proteins. PloS one. 2013;8(9). [PMID] [PMCID] [DOI:10.1371/journal.pone.0073957]

27. Doytchinova IA, Flower DR. VaxiJen: a server for prediction of protective antigens, tumour antigens and subunit vaccines. BMC Bioinform. 2007;8(1):4. [DOI:10.1186/1471-2105-8-4] [PMID] [PMCID]

28. Schneidman-Duhovny $D$, Inbar $Y$, Nussinov R, Wolfson HJ. PatchDock and SymmDock: servers for rigid and symmetric docking. Nucleic Acids Res. 2005;33(suppl_2):W363-W7. [DOI:10.1093/nar/gki481] [PMID] [PMCID]

29. Duhovny D, Nussinov R, Wolfson H. Efficient unbound docking of rigid molecules, p 185-200. Algorithms in bioinformatics Lecture notes in 
computer science. 2002;2452. [DOI:10.1007/3540-45784-4 14]

30. Afzali F, Ghahremanifard P, Ranjbar MM, Salimi M. Exploring PLAC1 Structure and Underlying Mechanisms to Design a Derivative Vaccine Against Breast Cancer Progression; In-Silico Study. Curr. Proteom. 2020;17(5):379-91. [DOI:10.2174/1570164617666191203111451]

31. Gasteiger E, Hoogland C, Gattiker A, Wilkins MR, Appel RD, Bairoch A. Protein identification and analysis tools on the ExPASy server. The proteomics protocols handbook: Springer; 2005. p. 571-607. [DOI:10.1385/1-59259-890-0:571]

32. Kelley LA, Mezulis S, Yates CM, Wass MN, Sternberg MJ. The Phyre2 web portal for protein modeling, prediction and analysis. Nat. Protoc. 2015;10(6):845. [DOI:10.1038/nprot.2015.053] [PMID] [PMCID]

33. Lovell SC, Davis IW, Arendall III WB, De Bakker PI, Word JM, Prisant MG, et al. Structure validation by $C \alpha$ geometry: $\varphi, \psi$ and $C \beta$ deviation. Proteins: Struct. Funct. Bioinform. 2003;50(3):437-50. [DOI:10.1002/prot.10286] [PMID]

34. Wiederstein M, Sippl MJ. ProSA-web: interactive web service for the recognition of errors in threedimensional structures of proteins. Nucleic Acids Res. 2007;35(suppl_2):W407-W10. [DOI:10.1093/nar/gkm290] [PMID] [PMCID]

35. Pierce BG, Wiehe K, Hwang H, Kim B-H, Vreven $\mathrm{T}$, Weng Z. ZDOCK server: interactive docking prediction of protein-protein complexes and symmetric multimers. Bioinformatics. 2014;30(12):1771-3. [PMID] [PMCID] [DOI:10.1093/bioinformatics/btu097]

36. Kozakov D, Hall DR, Xia B, Porter KA, Padhorny D, Yueh $C$, et al. The ClusPro web server for proteinprotein docking. Nat. Protoc. 2017;12(2):255. [DOI:10.1038/nprot.2016.169] [PMID] [PMCID]

37. López-Blanco JR, Aliaga JI, Quintana-Ortí ES, Chacón P. iMODS: internal coordinates normal mode analysis server. Nucleic Acids Res. 2014;42(W1):W271-W6. [DOI:10.1093/nar/gku339] [PMID] [PMCID]

38. Liu C, Zhou Q, Li Y, Garner LV, Watkins SP, Carter $\mathrm{L}$, et al. Research and development on therapeutic agents and vaccines for COVID-19 and related human coronavirus diseases. ACS Cent. Sci. 2020. [PMID] [PMCID] [DOI:10.1021/acscentsci.0c00272]

39. Kibria K, Ullah H, Miah M. The multi-epitope vaccine prediction to combat Pandemic SARS-
CoV-2, an immunoinformatic approach. Preprints 2020. [DOI:10.21203/rs.3.rs-21853/v1]

40. De Groot AS, Sbai H, Aubin CS, McMurry J, Martin W. Immuno-informatics: Mining genomes for vaccine components. Immunol. Cell Biol. 2002;80(3):255-69.

[DOI:10.1046/j.14401711.2002.01092.x] [PMID]

41. Purcell AW, McCluskey J, Rossjohn J. More than one reason to rethink the use of peptides in vaccine design. Nat. Rev. Drug Discov. 2007;6(5):404-14. [DOI:10.1038/nrd2224] [PMID]

42. Christie JM, Chapel H, Chapman RW, Rosenberg WM. Immune selection and genetic sequence variation in core and envelope regions of hepatitis C virus. Hepatology. 1999;30(4):103744. [DOI:10.1002/hep.510300403] [PMID]

43. Twiddy SS, Holmes EC, Rambaut A. Inferring the rate and time-scale of dengue virus evolution. Mol. Biol. Evol. 2003;20(1):122-9. [DOI:10.1093/molbev/msg010] [PMID]

44. Shen Z, Xiao Y, Kang L, Ma W, Shi L, Zhang L, et al. Genomic Diversity of Severe Acute Respiratory Syndrome-Coronavirus 2 in Patients With Coronavirus Disease 2019. Clin. Infect. Dis. 2020;71(15):713-20. [DOI:10.1093/cid/ciaa203] [PMID] [PMCID]

45. Lan J, Ge J, Yu J, Shan S, Zhou H, Fan S, et al. Structure of the SARS-CoV-2 spike receptorbinding domain bound to the ACE2 receptor. Nature. 2020;581(7807):215-20. [DOI:10.1038/s41586-020-2180-5] [PMID]

46. Cong $Y$, Ulasli $M$, Schepers $H$, Mauthe $M$, V'kovski $P$, Kriegenburg F, et al. Nucleocapsid protein recruitment to replication-transcription complexes plays a crucial role in coronaviral life cycle. J. Virol. 2020;94(4).

[DOI:10.1128/JVI.01925-19]

47. Leung DTM, Chi Hang TF, Chun Hung M, Sheung Chan PK, Cheung JLK, Niu H, et al. Antibody response of patients with severe acute respiratory syndrome (SARS) targets the viral nucleocapsid. The Journal of infectious diseases. 2004;190(2):379-86. [DOI:10.1086/422040] [PMID] [PMCID]

48. Kim PS, Reicin AS. Discontinuation of VIOXX. Lancet. 2005;365(9453):23. [DOI:10.1016/S01406736(04)17652-6]

49. Okada M, Takemoto $Y$, Okuno $Y$, Hashimoto S, Yoshida S, Fukunaga $Y$, et al. The development of vaccines against SARS corona virus in mice and SCID-PBL/hu mice. Vaccine. 2005;23(17- 
18):2269-72.

[PMID]

[DOI:10.1016/i.vaccine.2005.01.036]

50. Hofmeyr SA. An interpretative introduction to the immune system. Design principles for the immune system and other distributed autonomous systems. 2001;3:28-36.

51. Tritto E, Mosca F, De Gregorio E. Mechanism of action of licensed vaccine adjuvants. Vaccine. 2009;27(25-26):3331-4.

[DOI:10.1016/j.vaccine.2009.01.084] [PMID]

52. Buonaguro FM, Tornesello ML, Buonaguro L. New adjuvants in evolving vaccine strategies. Expert Opin Biol Ther. 2011;11(7):827-32. [DOI:10.1517/14712598.2011.587802] [PMID]

53. Yuzawa S, Kurita-Ochiai T, Hashizume T, Kobayashi R, Abiko Y, Yamamoto M. Sublingual vaccination with fusion protein consisting of the functional domain of hemagglutinin $A$ of Porphyromonas gingivalis and Escherichia coli maltose-binding protein elicits protective immunity in the oral cavity. FEMS Immunol Med Microbiol. 2012;64(2):265-72.

[DOI:10.1111/j.1574-695X.2011.00895.x] [PMID]

54. Fang F, Ma J, Ni W, Wang F, Sun X, Li Y, et al. MUC1 and maltose-binding protein recombinant fusion protein combined with Bacillus Calmette-Guerin induces MUC1-specific and nonspecific anti-tumor immunity in mice. Mol. Med. Rep. 2014;10(2):1056-64. [DOI:10.3892/mmr.2014.2306] [PMID]
55. Zhao X, Ma J, Fang F, Zhou J, Song X, Liu Z, et al. Effect of Escherichia coli maltose-binding protein on mouse Th1 cell activation. Chin. J. Microbiol. Immunol. 2009;25(6):504-7.

56. Fernandez $S$, Palmer DR, Simmons $M$, Sun $P$, Bisbing J, McClain S, et al. Potential role for Tolllike receptor 4 in mediating Escherichia coli maltose-binding protein activation of dendritic cells. Infect. Immun. 2007;75(3):1359-63. [DOI:10.1128/IAI.00486-06] [PMID] [PMCID]

57. Routzahn KM, Waugh DS. Differential effects of supplementary affinity tags on the solubility of MBP fusion proteins. J. Struct. Funct. Genomics. 2002;2(2):83-92. [DOI:10.1023/A:1020424023207] [PMID]

58. Chen X, Zaro JL, Shen W-C. Fusion protein linkers: property, design and functionality. Adv. Drug Deliv. Rev. 2013;65(10):1357-69. [PMID] [PMCID] [DOI:10.1016/j.addr.2012.09.039]

59. Terpe K. Overview of tag protein fusions: from molecular and biochemical fundamentals to commercial systems. Appl. Microbiol. Biotechnol. 2003;60(5):523-33. [DOI:10.1007/s00253-0021158-6] [PMID]

60. Jie J, Zhang $\mathrm{Y}$, Zhou H, Zhai X, Zhang N, Yuan H, et al. CpG ODN1826 as a promising mucin1maltose-binding protein vaccine adjuvant induced DC maturation and enhanced antitumor immunity. Int. J. Mol. Sci. 2018;19(3):920. [DOI:10.3390/ijms19030920] [PMID] [PMCID] 